\title{
On Conditional Expectation of Random Sets (*).
}

\author{
Midher Valadter (Montpellier, France)
}

\begin{abstract}
Summary. - Let $\Gamma$ be a random set with values in closed convex non empty subsets of the dual of a separable Fréchet space. Then its conditional expectation with respect to a sub-tribe is proved to exist and is related to regular conditional probability.
\end{abstract}

\section{Introduction.}

This paper intends to give new results in the field of conditional expectation of random sets. The framework is that of SAINTE-BEUVE [9]: the random sets are closed convex subsets of the dual space of a separable Fréchet space. The new points are:

1) the "separability" assumption on $(\Omega, \Re)$ is relaxed (in [9] theorem 6, p. $367 \mathfrak{B}$ is assumed to be the Borel tribe of a Suslin topology) and the expectation we obtain is smaller (in [9] it may happen that $\Gamma$ is $\mathfrak{B}$-measurable and $E^{\mathfrak{B}} \Gamma \supsetneqq \Gamma$, in the present paper $E^{\mathscr{S}} \Gamma$ equals $T$ );

2) the existence proof uses the notion of essential supremum of a family of multifunctions. This notion has been introduced by the author [10], [11];

3 ) the conditional expection is related to regular conditional probability (when it exists). Thus [16], theorem 9.1, p. 27 and [17], theorem A of $\S 26.1$, p. 354 are extended to multifunctions.

These results extends foregoing theorems of the author [12] and CASTAING-VALADIER [3] chapter VIII. Other references are [15], [1], [7], [4], [5], [6].

\section{1. - Prerequisites and notations.}

Let $E$ be a separable Fréchet space with a fundamental sequence of seminorms $\left(p_{n}\right)$. Let $V_{n}$ be the neighbourhood of $0: V_{n}=\left\{\dot{x} \mid p_{n}(x) \leqslant 1\right\}$.

The dual space $E^{\prime}$ with the weak topology $\sigma\left(E^{\prime}, E\right)$ is. denoted $E_{s}^{\prime}$. It is the union of the $V_{n}^{0}$ (the polar set of $V_{n}$ ) which are compact metrizable. Hence $E_{s}^{\prime}$ is a Lusin space. We denote by $\mathfrak{B}\left(E_{s}^{\prime}\right)$ its Borel tribe.

(*) Entrata in Redazione il 25 settembre 1979.

6 - Annali ai Matematica 
Now let $(\Omega, \mathcal{A}, P)$ be a probability space and $\mathcal{B}$ a sub tribe of $\mathcal{A}$. We denote $\mathfrak{L}_{E^{\prime}}^{1}(\Omega, \mathcal{A}, P)$ (shortly $\mathfrak{L}_{E^{\prime}}^{1}$ or $\mathfrak{L}_{E^{\prime}}^{1}(\mathfrak{A})$ to specify the tribe) the space of «integrable " functions from $\Omega$ to $E^{\prime}$ (see [9] for a detailed study). Namely $u \in \mathcal{L}_{E^{\prime}}^{1}$ if

1) $u$ is $\mathcal{A}-\mathfrak{B}\left(E_{s}^{\prime}\right)$ measurable (see the appendix for equivalent properties);

2) $\exists \alpha \in \mathfrak{L}_{R}^{1}$ and $\exists n \in N$ such that $\alpha \geqslant 0$ and $u(\omega) \in \alpha(\omega) V_{n}^{0}$ a.e.

We denote $\mathfrak{L}_{E}^{\infty}\left(\Omega, \mathcal{A}, P\right.$ ) (shortly $\mathfrak{L}_{E}^{\infty}$ or $\mathfrak{L}_{E}^{\infty}(\mathfrak{A})$ ) the space of all measurable bounded function $v: \Omega \rightarrow E$. Such $a v$ is limit of a sequence of measurable bounded functions assuming a countable number of values.

The space $\mathfrak{L}_{E^{\prime}}^{1}$ and $\mathfrak{L}_{s}^{\infty}$ are in duality and are decomposable ([3] VII.3, p. 197). We shall often use the quotient spaces $L_{E^{\prime}}^{1}$ and $L_{z}^{\infty}$ which are in separated duality.

So we have three pairs of spaces in duality: $\left(E, E^{\prime}\right),\left(L_{E^{\prime}}^{1}(\mathcal{A}), L_{\mathcal{E}}^{\infty}(\mathcal{A})\right)$ and $\left(L_{Z^{\prime}}^{1}(\mathfrak{B})\right.$, $\left.L_{E}^{\infty}(\mathcal{B})\right)$. In any of these pairs the bilinear form will be denoted $\langle\cdot, \cdot\rangle$, the indicator function of a set $C$ will be denoted $\delta(\cdot \mid C)$ and its support function $\delta^{*}(\cdot \mid C)$.

If $\Gamma$ is a multifunction from $\Omega$ to $E^{\prime}$ we denote by $\mathfrak{L}_{\Gamma}^{1}(\mathcal{A})$ the set $\left\{u \in \mathfrak{L}_{E^{\prime}}^{1}(\mathcal{A}) \mid u(\omega) \in\right.$ $\in \Gamma(\omega)$ a.e. $\}$ and $L_{\Gamma}^{1}(\mathcal{A})$ the corresponding space of equivalence classes. We say that $\Gamma$ is $\mathcal{A}$-measurable or is a $\mathcal{A}$-random set if its graph $G(\Gamma)$ belongs to $\mathcal{A} \otimes \mathfrak{B}\left(E_{\mathrm{s}}^{\prime}\right)$. Application of some theorems about measurable multifunctions will give $\hat{A}$-random set ( $\hat{\mathfrak{A}}$ is the completion of $\mathfrak{A}$ ) but the appendix shows the existence of an equivalent (for equality almost everywhere) $t$-random set.

We use the following symbols:

$\operatorname{CRS}\left(\mathfrak{A} ; E^{\prime}\right)$ denotes the set of closed non empty $\mathcal{A}$-random subsets of $E^{\prime}$;

$\operatorname{CRS}\left(A ; V_{n}^{0}\right)$ denotes those which are contained in $V_{n}^{0}$, and we add one $C$ to mean that convexity is assumed: $\operatorname{CCRS}\left(\mathfrak{A} ; E^{\prime}\right)$ etc.....

THeOReM 1. - Let $\left(\Gamma_{i}\right)_{i \in I}$ be a family in $\mathrm{CRS}\left(\mathcal{A} ; V_{n}^{0}\right)$, then it admits an essential supremum $\Gamma$ in $\operatorname{CRS}\left(\mathfrak{A} ; V_{n}^{0}\right)$ : that is

1) $\forall i, \Gamma_{i}(\omega) \subset \Gamma(\omega)$ a.e.;

2) if $\Sigma \in \operatorname{CRS}\left(\mathcal{A} ; \nabla_{n}^{0}\right)$ and $\forall i, \Gamma_{i}(\omega) \subset \Sigma(\omega)$ a.e., then $\Gamma(\omega) \subset \Sigma(\omega)$ a.e.

Moreover there exists a countable subfamily $\left(\Gamma_{i}\right)_{i \in D}$ fuch that $\Gamma(\omega)=\mathrm{cl} \bigcup_{i \in D} \Gamma_{i}(\omega)$ a.e. (where el abbreviates closure).

For the proof we refer to VALADIER $[10,11]$ (prop. 1.14).

\section{REMARKS:}

1) In $[10,11]$ we use the measurability property " $\left\{\omega \mid \Gamma_{i}(\omega) \cap \vartheta \neq \emptyset\right\} \in \mathcal{A}$ for every open set $\mathcal{V}$ ». Here this is verified with $\hat{\mathfrak{A}}$ : see appendix. 
2) In $[10,11]$ the result is stated with a separable metrizable space in place of $V_{n}^{0}$. If $E$ is a separable reflexive Banach space, the theorem can be stated for a family in $\operatorname{CRS}\left(\mathcal{A} ; E^{\prime}\right)$.

THEOREM 2. - There exists a linear mapping

$$
E^{\mathcal{S}}: L_{E^{\prime}}^{1}(\mathcal{A}) \rightarrow L_{R^{\prime}}^{1}(\mathfrak{B}) \quad \text { such that } \forall u \in L_{\mathbb{H}^{\prime}}^{\mathfrak{1}}(\mathcal{A}), \quad \forall B \in \mathcal{B}, \quad \int_{B} u d P=\int_{B} E^{\mathfrak{S}}(u) d P .
$$

Reference: SAINTE-BEUVE [9], prop. 7, p. 366.

We shall still denote $E^{\mathfrak{S}}(f)$ for 2 - scalar integrable (or quasi-integrable) function $f$.

LEMrVA 1. - If $u \in L_{\mathbb{E}^{\prime}}^{1}(\mathfrak{A})$ and $v \in L_{\tilde{z}}^{\infty}(\mathfrak{B})$ one has $E^{\mathscr{S}}(\langle v(\cdot), u(\cdot)\rangle)(\omega)=\left\langle v(\omega), E^{\mathscr{S}}(u)(\omega)\right\rangle$ a.e. and $\int_{\Omega}\langle v, u\rangle d P=\int_{\Omega}\left\langle v, E^{\mathfrak{B}}(u)\right\rangle d P$.

Proof. - It suffices to prove the last formula. Indeed if it holds for $v \chi_{B}(B \in \mathcal{B})$ then $\int_{B}\langle v, u\rangle d P=\int_{B}\left\langle v, E^{\mathscr{B}_{3}}(u)\right\rangle d P$ hence $\int_{B} E^{\mathscr{S}_{\mathcal{B}}}(\langle v, u\rangle) d P=\int_{B}\left\langle v, E^{\mathscr{S}_{3}}(u)\right\rangle d P$ which is equivalent to $E^{\mathscr{S}_{\mathcal{B}}}(\langle v, u\rangle)=\left\langle v, E^{\mathcal{S}_{3}}(u)\right\rangle$.

The equality $\int_{\Omega}\langle v, u\rangle d P=\int_{\Omega}\left\langle v, E^{\mathscr{S}}(u)\right\rangle d P$ holds if $v$ assumes a countable number of values. By a limiting process the equality is valid for any $v$.

\section{2. - Preliminary version of the main theorem.}

LEMma 2. - Let $\Gamma \in \operatorname{CCRS}\left(\mathcal{A} ; V_{n}^{0}\right)$. There exists $\Sigma \in \operatorname{CCRS}\left(\mathscr{B} ; V_{n}^{0}\right)$ such that

1) $\Sigma$ is the smallest (for inclusion a.e.) of the $\Theta \in \operatorname{CRS}\left(\mathscr{B} ; V_{n}^{0}\right)$ such that

$$
\forall u \in L_{\Gamma}^{1}(\mathcal{A}), \quad E^{\mathfrak{A}}(u)(\omega) \in \Theta(\omega) \text { a.e. }
$$

2) $\Sigma$ is the unique $\Theta \in \operatorname{CCRS}\left(\mathscr{B} ; V_{n}^{0}\right)$ such that $\forall v \in L_{E}^{\infty}(\mathcal{B})$,

$$
\int_{\Omega} \delta^{*}(v(\omega) \mid \Gamma(\omega)) P(d \omega)=\int_{\Omega} \delta^{*}(v(\omega) \mid \Theta(\omega)) P(d \omega)
$$

REMark. - Assertion 1) means that $\Sigma$ is the essential supremum of the family of multifunction $\left(\omega \mapsto\left\{E^{\mathcal{B}}(u)(\omega)\right\}\right), u \in L_{\Gamma}^{1}(\mathcal{A})$.

Proof. - 1) Let $\Sigma$ be the essential supremum of the $\omega \mapsto\left\{E^{\mathscr{S}}(u)(\omega)\right\}$. By theorem 1 there exists a sequence $\left(u_{n}\right)$ in $L_{\Gamma}^{1}(\mathcal{A})$ such that $\Sigma(\omega)=\operatorname{cl} \bigcup_{n}\left\{E^{\mathscr{A}}\left(u_{n}\right)(\omega)\right\}$.

Consider the countable family $\left(u_{i}^{\prime}\right)_{i \in D}$ of barycenters with rational coefficients of all finite subsets of $\left\{u_{n} \mid n \in N\right\}$. Each $u_{i}^{\prime}$ belongs to $L_{T}^{1}(\mathcal{A})$. 
So

$$
\Sigma(\omega) \ni E^{\mathscr{\beta}}\left(u_{i}^{\prime}\right)(\omega) \quad \text { a.e. }
$$

and

$$
\Sigma(\omega)=\mathbf{c l} \bigcup_{i \in D}\left\{E^{\Re}\left(u_{i}^{\prime}\right)(\omega)\right\} \quad \text { a.e. }
$$

That proves the convexity of $\Sigma(\omega)$.

2) a) We begin to prove that $\Sigma$ verifies

$$
\delta^{*}(x \mid \Sigma(\omega))=\mathbb{E}^{\mathscr{S}}\left[\delta^{*}(x \mid \Gamma(\cdot))\right](\omega)
$$

One has

$$
\begin{aligned}
\delta^{*}(x \mid \Sigma(\omega)) & =\sup _{n}\left\langle x, E^{\mathscr{S}}\left(u_{n}\right)(\omega)\right\rangle \\
& =\sup _{n} E^{\mathscr{S}}\left[\left\langle x, u_{n}(\cdot)\right\rangle\right](\omega) \\
& \leqslant E^{\mathscr{S}}\left[\delta^{*}(x \mid \Gamma(\cdot))\right](\omega) .
\end{aligned}
$$

Conversely, for every $n$ there exists (thanks to [3] III.22) $f_{n} \in \mathfrak{L}_{\Gamma}^{1}(\mathfrak{A})$ such that

$$
\left\langle x, f_{n}(\omega)\right\rangle \geqslant \delta^{*}(x \mid \Gamma(\omega))-\frac{1}{n} \quad \text { a.e. }
$$

Then for every $B \in \mathfrak{B}$

(1)

$$
\int_{B}\left\langle x, f_{n}\right\rangle d P \rightarrow \int_{B} \delta^{*}(x \mid \Gamma) d P
$$

As $E^{\mathfrak{S}}\left(f_{n}\right)(\omega) \in \Sigma(\omega)$ a.e. one has

$$
\begin{aligned}
\int_{B}\left\langle x, f_{n}\right\rangle d P & =\int_{B}\left\langle x, E^{\mathcal{S}_{3}}\left(f_{n}\right)\right\rangle d P \\
& \leqslant \delta^{*}(x \mid \Sigma) d P
\end{aligned}
$$

which combined with (1) gives

$$
\int_{B} \delta^{*}(x \mid \Gamma) d P \leqslant \int_{B} \delta^{*}(x \mid \Sigma) d P
$$

hence

$$
E^{\mathfrak{B}}\left[\delta^{*}(x \mid \Gamma(\cdot))\right](\omega) \leqslant \delta^{*}(x \mid \Sigma(\omega)) \quad \text { a.e. }
$$


b) We turn now to prove, $\forall v \in L_{z}^{\infty}(\mathfrak{B})$

$$
\int_{\Omega} \delta^{*}(v \mid \Gamma) d P=\int_{\Omega} \delta^{*}(v \mid \Sigma) d P
$$

If $v=\chi_{B} x$ that follows from $a$ ). If $v$ assumes a countable number of values, $v=\Sigma_{\chi_{B_{n}}} x_{n}$ (the $B_{n}$ disjointed), then for every $n$,

$$
\int_{B_{n}} \delta^{*}\left(x_{n} \mid \Gamma\right) d P=\int_{B_{n}} \delta^{*}\left(x_{n} \mid \Sigma\right) d P .
$$

The sum gives the expected equality.

Now if $v=\lim v_{k}$ where $v_{k}$ assumes a countable number of values and the limit is uniform, one has

$$
\left|\delta^{*}(v(\omega) \mid \Gamma(\omega))-\delta^{*}\left(v_{k}(\omega) \mid \Gamma(\omega)\right)\right| \leqslant p_{n}\left(v(\omega)-v_{k}(\omega)\right)
$$

and the same inequality with $\Sigma$ in place of $\Gamma$.

Thus

$$
\begin{aligned}
\int \delta^{*}(v \mid \Gamma) d P & =\lim \int \delta^{*}\left(v_{k} \mid \Gamma\right) d P \\
& =\lim \int \delta^{*}\left(v_{k} \mid \Sigma\right) d P \\
& =\int \delta^{*}(v \mid \Sigma) d P
\end{aligned}
$$

c) It remains to prove uniqueness. Let $\Theta_{1}$ and $\Theta_{2}$ in $\operatorname{CORS}\left(\mathscr{B} ; V_{n}^{0}\right)$ such that for every $v \in L_{B}^{\infty}(\Re)$

$$
\int \delta^{*}\left(v \mid \Theta_{i}\right) d P=\int \delta^{*}(v \mid \Gamma) d P
$$

By [3] (VII.7, p. 200) the polar of

$$
\begin{aligned}
v & \mapsto \int \delta^{*}\left(v \mid \Theta_{i}\right) d P \quad \text { is } \\
u & \mapsto \int \delta\left(u \mid \Theta_{i}\right) d P=\delta\left(u \mid L_{\Theta_{i}}^{1}(\mathcal{B})\right) .
\end{aligned}
$$

Thus $L_{\Theta_{1}}^{1}(\mathscr{B})=L_{\Theta_{2}}^{1}(\mathscr{B})$. This set is non empty (because $=L_{\Sigma}^{1}(\mathscr{B})$ ). By standard arguments (see for example [13] Séminaire d'Analyse Convexe, lemma 5) $\Theta_{1}(\omega)=$ $=\Theta_{2}(\omega)$ a.e.

\section{3. - The main theorem.}

In lemma 2 we obtained the conditional expectation $\Sigma$ of $\Gamma \in \operatorname{CCRS}\left(\mathfrak{A} ; V_{n}^{0}\right)$. We turn now to $\Gamma \in \operatorname{CCRS}\left(\mathfrak{A} ; E^{\prime}\right)$. 
THEOREM 3. - Let $\Gamma \in \operatorname{CCRS}\left(\mathfrak{A} ; E^{\prime}\right)$ such that $L_{\Gamma}^{1}(\mathfrak{A}) \neq \emptyset$. There exists $\Sigma \in \operatorname{CCRS}\left(\mathfrak{B} ; E^{\prime}\right)$ such that:

1) $\Sigma$ is the smallest of the $\Theta \in \operatorname{CRS}\left(\Re ; E^{\prime}\right)$ such that $\forall u \in L_{\Gamma}^{1}(\mathcal{A}), E^{\mathscr{S}}(u)(\omega) \in$ $\in \Theta(\omega)$ a.e.

2) $\Sigma$ is the unique $\Theta$ in $\operatorname{CCRS}\left(\mathfrak{B} ; E^{\prime}\right)$ such that $\forall v \in L_{z}^{\infty}(\mathcal{B})$,

$$
\int \delta^{*}(v \mid \Gamma) d P=\int \delta^{*}(v \mid \Theta) d P
$$

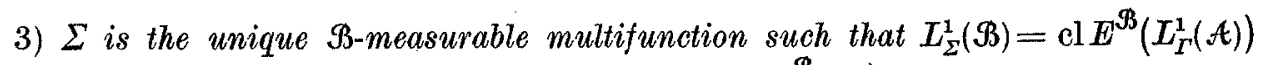
where cl denotes the closure with respect to $\sigma\left(L_{E^{\prime}}^{1}(\mathfrak{B}), L_{E}^{\mathscr{B}}\left(\mathscr{B}_{3}\right)\right)$.

4) if there exists a regular conditional probability $P^{\mathcal{S}_{3}}(\cdot \mid \cdot)$ on $(\Omega, \mathcal{A})$, then for $P$-almost every $\omega, \mathfrak{L}_{\Gamma}^{1}\left(\Omega, \mathcal{A}, P^{\mathfrak{B}}(\cdot \mid \omega)\right)$ is $\neq \emptyset$ and

$$
\Sigma(\omega)=\operatorname{cl}\left\{\int u\left(\omega^{\prime}\right) P^{\mathscr{B}}\left(d \omega^{\prime} \mid \omega\right) \mid u \in \mathfrak{L}_{\Gamma}^{1}\left(\Omega, \mathcal{A}, P^{\mathfrak{B}_{3}}(\cdot \mid \omega)\right)\right\} .
$$

REMARK. - A regular conditional probability is a function $P^{\mathfrak{S}}(\cdot \mid \cdot): \mathcal{A} \times \Omega \rightarrow[0,1]$ such that

1) $\forall \omega, P^{\mathscr{S}}(\cdot \mid \omega)$ is a probability over $\mathcal{A}$,

2) $\forall A \in \mathcal{A}, P^{\mathfrak{S}}(A \mid \cdot)$ is $\mathfrak{B}$-measurable,

(2) 3) $\forall A \in \mathfrak{A}, \quad \forall B \in \mathfrak{B}, P(A \cap B) \int_{B} P^{\mathfrak{S}}(A \mid \omega) P(d \omega)$.

Formula (2) extends in the following way: for every $\varphi: \Omega \times \Omega \rightarrow[0, \infty]$ which is $\mathfrak{B} \otimes \mathfrak{A}$-measurable

$$
\int_{\Omega} \varphi(\omega, \omega) P(d \omega)=\int_{\Omega}\left[\int_{\Omega} \varphi\left(\omega, \omega^{\prime}\right) P^{\Re}\left(d \omega^{\prime} \mid \omega\right)\right] P(d \omega)
$$

Let $Q$ be the probability on $(\Omega \times \Omega, \mathscr{B} \otimes \mathfrak{A})$ which is the image of $P$ by $\omega \mapsto(\omega, \omega)$. Then $P^{\mathcal{S}_{3}}(\cdot \mid \cdot)$ is the disintegration of $Q$ with respect to the projection on $(\Omega, \mathcal{B})$. Such a disintegration exists when $t$ is the Borel tribe of a Suslin topology (Jirina's theorem). Formula (3) holds also for $\varphi \in \mathfrak{L}^{1}(\Omega \times \Omega, \mathfrak{B} \otimes \mathfrak{t}, Q)$.

Proof. - $A$ ) We suppose first that $0 \in \Gamma(\omega)$ a.e. We define $\Gamma_{n} \in \operatorname{CORS}\left(\mathcal{A} ; V_{n}^{0}\right)$ by $\Gamma_{n}(\omega)=\Gamma(\omega) \cap V_{n}^{0}$. Let $\Sigma_{n}$ be the conditional expectation of $\Gamma_{n}$ given by lemma 2 . It is clear that $\Sigma_{n}(\omega) \subset \Sigma_{n+1}(\omega)$ a.e.

We put $\Sigma(\omega)=\operatorname{cl}\left(\bigcup_{n} \Sigma_{n}(\omega)\right)$. It is a closed convex set containing 0 . Thanks to the formula

$$
\delta(x \mid \Sigma(\omega))=\left[\sup _{n} \delta^{*}\left(\cdot \mid \Sigma_{n}(\omega)\right)\right]^{*}(x)
$$


and the result of [3] (ch. VII) one has

$$
G(\Sigma) \in \widehat{\mathfrak{B}} \otimes \mathfrak{B}\left(E_{s}^{\prime}\right) .
$$

Using the appendix we may suppose that $\Sigma$ is $\mathscr{B}$-measurable.

B) Proof of 2).

a) First we suppose $0 \in \Gamma(\omega)$ a.e.

By Fatou lemma the formula valid for $\Gamma_{n}$ and $\Sigma_{n}$ remains valid for $\Gamma$ and $\Sigma:$

$$
\int \delta^{*}(v \mid \Gamma) d P=\int \delta^{*}(v \mid \Sigma) d P
$$

b) Now we relax the hypothesis $0 \in \Gamma(\omega)$. Let $u_{0} \in \mathfrak{L}_{\Gamma}^{\mathbf{1}}(\mathfrak{A})$. Put $\Gamma^{\prime}(\omega)=\Gamma(\omega)-$ $-u_{0}(\omega)$. The bijection $\left(\omega, x^{\prime}\right) \mapsto\left(\omega, x^{\prime}-u_{0}(\omega)\right)$ (which maps $\Omega \times E^{\prime}$ into itself) transforms $G(\Gamma)$ into $G\left(\Gamma^{\prime}\right)$, so $G\left(\Gamma^{\prime}\right) \in \mathcal{A} \otimes \Re\left(E_{s}^{\prime}\right)$. Let $\Sigma^{\prime}$ be the conditional expectation of $\Gamma^{\prime}$ and $\Sigma(\omega)=\Sigma^{\prime}(\omega)+E^{\mathcal{B}}\left(u_{0}\right)(\omega)$. The foregoing argument applies to $\Sigma^{\prime}$ and $\Sigma: \Sigma$ is measurable. At last, if $v \in L_{E}^{\infty}(\mathcal{B})$,

$$
\begin{aligned}
& \delta^{*}(v \mid \Gamma)=\delta^{*}\left(v \mid \Gamma^{\prime}\right)+\left\langle v, u_{0}\right\rangle \\
& \delta^{*}(v \mid \Sigma)=\delta^{*}\left(v \mid \Sigma^{\prime}\right)+\left\langle v, E^{\Re}\left(u_{0}\right)\right\rangle .
\end{aligned}
$$

Then, thanks to $a$ ) and lemma 1), $\int \delta^{*}(v \mid \Gamma) d P=\int \delta^{*}(v \mid \Sigma) d P$.

The uniqueness is proved as in lemma 2.

C) PRoof of 1).

First we prove $u \in L_{\Gamma}^{1}(\mathcal{A}) \Rightarrow E^{\mathscr{B}}(u) \in L_{\Sigma}^{1}(\mathfrak{B})$.

One has for $v \in L_{E}^{\infty}(\mathfrak{B})$,

$$
\begin{aligned}
\left\langle v, E^{\aleph}(u)\right\rangle & =\int\langle v, u\rangle d P \quad(\text { lemma 1) } \\
& \leqslant \int \delta^{*}(v \mid \Gamma) d P \\
& =\int \delta^{*}(v \mid \Sigma) d P .
\end{aligned}
$$

Then, with the polar functionals, for every $u^{\prime} \in L_{E^{\prime}}^{\mathbf{1}}(\mathfrak{B})$

$$
\delta\left(u^{\prime} \mid\left\{E^{\mathfrak{B}}(u)\right\}\right) \geqslant \int \delta\left(u^{\prime} \mid \Sigma\right) d P .
$$

Taking $u^{\prime}=E^{\mathfrak{B}}(u)$ that proves $E^{\mathscr{S}}(u)(\omega) \in \Sigma^{\prime}(\omega)$ a.e.

It remains to prove that if $\Theta(\omega) \ni E^{\mathcal{S}_{3}}(u)(\omega)$ a.e. for every $u \in L_{\Gamma}^{1}(\mathcal{A})$, then $\Theta(\omega) \supset$ $\supset \Sigma(\omega)$ a.e. Let $u_{0} \in \mathfrak{C}_{\Gamma}^{1}(\mathcal{A})$. One has $\Theta(\omega)-E^{\mathfrak{S}}\left(u_{0}\right)(\omega) \ni E^{\mathfrak{B}}(u)(\omega)-E^{\mathfrak{B}^{3}}\left(u_{0}\right)(\omega)$. Hence $\Theta(\omega)-E^{\mathfrak{S}_{3}}\left(u_{0}\right)(\omega)$ contains all the $E^{\mathcal{B}}\left(u^{\prime}\right)$ where $u^{\prime} \in L_{\Gamma_{n}^{\prime}}^{1}(\mathcal{A})$, so it contains $\Sigma_{n}^{\prime}(\omega)$.

Hence $\Theta(\omega)-E^{\mathcal{B}^{3}}\left(u_{0}\right)(\omega) \supset \Sigma^{\prime}(\omega)=\Sigma(\omega)-E^{\mathcal{S}}\left(u_{0}\right)(\omega)$. 
D) Proof of 3)

By 1) we have $L_{\Sigma}^{1}(\mathscr{B}) \supset E^{\mathfrak{B}}\left(L_{T}^{1}(\mathscr{A})\right)$. Moreover $L_{\Sigma}^{1}(\mathscr{B})$ is $\sigma\left(L_{\xi^{\prime}}^{1}(\mathfrak{B}), L_{E}^{\infty}(\mathfrak{B})\right)$ closed because its indicator function is the polar of the functional

$$
v \mapsto \int \delta^{*}(v \mid \Sigma) d P
$$

It remains to prove

$$
\delta^{*}\left(v \mid L_{\Sigma}^{1}(\mathfrak{B})\right)=\delta^{*}\left(v \mid E^{\mathfrak{B}}\left(L_{\Gamma}^{1}(\mathcal{A})\right)\right)
$$

One has

$$
\begin{aligned}
\delta^{*}\left(v \mid E^{\mathfrak{S}}\left(L_{\Gamma}^{1}(\mathcal{A})\right)\right) & =\sup \left\{\left\langle v, E^{\mathfrak{S}}(u)\right\rangle \mid u \in L_{\Gamma}^{1}(\mathcal{A})\right\} \\
& =\sup \left\{\langle v, u\rangle \mid u \in L_{\Gamma}^{1}(\mathcal{A})\right\} \quad(\text { lemma 1) } \\
& =\delta^{*}\left(v \mid L_{\Gamma}^{1}(\mathcal{A})\right) \\
& =\int \delta^{*}(v \mid \Gamma) d P \\
& =\int \delta^{*}(v \mid \Sigma) d P \\
& =\delta^{*}\left(v \mid L_{\Sigma}^{1}(\mathfrak{S})\right) .
\end{aligned}
$$

At last uniqueness has still be noticed.

E) Proof of 4)

Let $u_{0} \in \mathcal{L}_{\Sigma}^{1}(\mathcal{A})$. There exist $\alpha \in \mathcal{L}_{\boldsymbol{R}}^{1}$ and $n \in N$ such that $\alpha \geqslant 0$ and $u_{0}(\omega) \in \alpha(\omega) V_{n}^{0}$ a.e. Applying formula $(3)$ to $\varphi\left(\omega, \omega^{\prime}\right)=\alpha\left(\omega^{\prime}\right)$, one obtains

$$
\int_{\Omega} \alpha d P=\int_{\Omega}\left[\int_{\Omega} \alpha\left(\omega^{\prime}\right) P^{\$}\left(d \omega^{\prime} \mid \omega\right)\right] P(d \omega) .
$$

Hence for $P$-almost every $\omega$ (that is except if $\omega \in N, N$ negligible) $\alpha$ is $P^{\mathfrak{B}}(\cdot \mid \omega)$ integrable and so $u_{0} \in \mathcal{L}_{E^{\prime}}^{\mathbb{I}}\left(\Omega, \mathcal{A}, P^{\mathfrak{B}}(\cdot \mid \omega)\right.$ ) (remark: if $u_{0}(\omega) \in \Gamma(\omega)$ if $\omega \notin N^{\prime}$ where $P\left(N^{\prime}\right)=0$, then for $P$-almost every $\omega, N^{\prime}$ is $P^{\mathfrak{B}}(\cdot \mid \omega)$ negligible $)$.

Put for $\omega \notin N$

$$
\Theta(\omega)=\operatorname{cl}\left\{\int u\left(\omega^{\prime}\right) P^{\Re}\left(d \omega^{\prime} \mid \omega\right) \mid u \in \mathbb{L}_{\Gamma}^{\perp}\left(\Omega, \mathcal{A}, P^{\Re}(\cdot \mid \omega)\right)\right\}
$$

It is a closed convex non empty subset of $E^{\prime}$.

For $x \in E$

$$
\begin{aligned}
\delta^{*}(x \mid \Theta(\omega)) & =\sup \left\{\int\left\langle u\left(\omega^{\prime}\right), x\right\rangle P^{\mathfrak{B}}\left(d \omega^{\prime} \mid \omega\right) \mid u \in \mathbb{L}_{\Gamma}^{1}\left(\Omega, \mathcal{A}, P^{\mathfrak{B}}(\cdot \mid \omega)\right)\right\} \\
& =\int \delta^{*}\left(x \mid \Gamma\left(\omega^{\prime}\right)\right) P^{\mathfrak{B}}\left(d \omega^{\prime} \mid \omega\right)
\end{aligned}
$$

So $(\omega, x) \mapsto \delta^{*}(x \mid \Theta(\omega))$ is an integrand over $(\Omega, \mathcal{B}) \times E$. 
For $v \in L_{E}^{\infty}(\Re)$, one has

$$
\begin{aligned}
\int_{\Omega} \delta^{*}(v(\omega) \mid \Theta(\omega)) P(d \omega) & =\int_{\Omega}\left[\int_{\Omega} \delta^{*}\left(v(\omega) \mid \Gamma\left(\omega^{\prime}\right) P^{\Re}\left(d \omega^{\prime} \mid \omega\right)\right] P(d \omega)\right. \\
& =\int_{\Omega} \delta^{*}(v(\omega) \mid \Gamma(\omega)) P(d \omega)
\end{aligned}
$$

(formula (3) applied to $\varphi\left(\omega, \omega^{\prime}\right)=\delta^{*}\left(v(\omega) \mid \Gamma\left(\omega^{\prime}\right)\right)$ ).

By 2) $\Theta=\Sigma$ a.e.

Notation. - We shall denote $E^{\mathfrak{S}}(T)$ in place of $\Sigma$.

\section{COROLLARY:}

1) $\Gamma_{1} \subset \Gamma_{2}$ implies $E^{\mathfrak{B}}\left(\Gamma_{1}\right) \subset E^{\mathfrak{B}}\left(\Gamma_{2}\right)$.

2) if $\Gamma$ is $\mathfrak{B}_{3}$-measurable, $E^{\mathfrak{S}}(\Gamma)=\Gamma$ a.e.

3) if $\mathcal{C}$ is a sub-tribe of $\mathfrak{B}, E^{\mathrm{C}}(\Gamma)=E^{\mathcal{C}}\left(E^{\mathcal{A}}(\Gamma)\right)$.

4) if $\Sigma=E^{\mathfrak{B}}(I)$, for every $B \in \mathfrak{B}$ :

$$
\operatorname{cl}\left\{\int_{B} u d P \mid u \in L_{\Gamma}^{1}(\mathcal{A})\right\}=\operatorname{cl}\left\{\int_{B} u d P \mid u \in L_{\Sigma}^{1}(\mathcal{B})\right\}=\operatorname{cl}\left\{\int_{B} u d P \mid u \in L_{\Sigma}^{1}(\mathcal{A})\right\} .
$$

\section{ProOF :}

1) follows from 1) of the theorem;

2) follows from 2) of the theorem;

3) follows easily from 2) of the theorem;

4) follows from the calculus of the support functions.

\section{4. - Further remarks.}

1) Part 2) of theorem 3 implies

$$
\forall x \in E \quad \forall B \in \mathcal{B}, \quad \int_{B} \delta^{*}(x \mid \Gamma(\omega)) P(d \omega)=\int_{B} \delta^{*}(x \mid \Sigma(\omega)) P(d \omega),
$$

hence $\forall x \in E, \delta^{*}(x \mid \Sigma(\omega))=E^{\Re}\left[\delta^{*}(x \mid \Gamma(\cdot))\right](\omega)$ a.e.

But this condition does not characterize $\Sigma$. It gives uniqueness if $\Sigma(\omega)$ is supposed to be a closed convex weakly locally compact set which contains no line (see [3], III.35, p. 83). 
We give a counter example: let $\Omega=[0,1], \mathfrak{t}=\mathfrak{B}=\mathscr{B}([0,1]), P$ the Lebesgue measure, $E=\boldsymbol{R}^{2}, \Sigma(\omega)=\left\{(x, y) \in R^{2} \mid y=\omega x\right\}, \Sigma^{\prime}(\omega)=\boldsymbol{R}^{2}$.

It is easy to see that

$$
\int_{B} \delta^{*}(x \mid \Sigma(\omega)) P(d \omega)=\int_{B} \delta^{*}\left(x \mid \Sigma^{\prime}(\omega)\right) P(d \omega)
$$

(the integrals are $+\infty$ as soon as $x \neq 0$ and $P(B)>0$ ).

2) We give now an example which shows that the conditional expectation of [9] is bigger than ours. Let $\Omega=[0,1], \mathcal{A}=\mathfrak{B}=\mathfrak{B}([0,1]), P$ the Lebesgue measure, $E=R, K$ a Cantor like set with $P(K)>0$,

$$
\begin{aligned}
& \Gamma(\omega)=[0, \infty] \quad \text { if } \quad \omega \notin K \\
& =\{0\} \quad \text { if } \quad \omega \in K \text {. }
\end{aligned}
$$

Then, as it is said in OLECH [8] (p. 622) (see also [13]) the $\sigma\left(L^{1}, \mathfrak{C}\right)$ closure of $L_{T}^{1}$ is $L_{\Sigma}^{1}$ with $\Sigma(\omega)=[0, \infty[$. So the Sainte-Beuve's conditional expectation of $T$ is $\Sigma$.

3) It is easy to see that the conditional expectation of HIAI and UMEGAKI [6] can be defined as the essential supremum of $\left\{E^{\mathscr{S}}(u) \mid u \in L_{\Gamma}^{1}(\mathcal{A})\right\}$ (see [14] for more details).

4) In [2] (th. 3, p. 9-10) Castaing obtains a weakly compact valued conditional expectation. Namely let $F$ be a separable Banach space, $F_{b}^{\prime}$ its strong dual which is assumed separable, $T$ a multifunction with closed eonvex weakly compact values in $F$. Moreover $\exists \alpha \in \mathfrak{L}_{R}^{1}$ such that $\Gamma(\omega) \subset \alpha(\omega) B$ where $B$ denotes the unit ball of $F$.

We can apply theorem 3 with $E=F^{\prime}, E^{\prime}=F^{\prime \prime}$. It gives $\Sigma(\omega)=E^{S_{B}}(\Gamma)(\omega) \subset F^{\prime \prime}$ with $\Sigma(\omega) \subset E^{\mathfrak{B}}(\alpha) B^{\prime \prime}$ where $B^{\prime \prime}$ denotes the unit ball of $E^{\prime \prime}$.

As $L_{\Gamma}^{1}$ is $\sigma\left(L_{E}^{1}, L_{E}^{\infty}\right)$ compact ([2] theorem 1) $L_{\Sigma}^{1}$ is equal to $E^{\mathscr{S}^{3}}\left(L_{\Gamma}^{1}\right)$ hence contained in $L_{F}^{1}$. The inclusion $L_{\Sigma}^{1} \subset L_{F}^{1}$ implies $\Sigma(\omega) \subset F$ a.e.

\section{5. - Appendix.}

1) Let $u: Q \rightarrow E_{s}^{\prime}$. There are many equivalent measurability properties. It is well known that " $u$ is $\mathcal{A}-\mathscr{B}\left(E_{s}^{\prime}\right)$ measurable" is equivalent to " $u$ is scalarly measurable ", that is $\forall x \in E, \omega \mapsto\langle u(\omega), x\rangle$ is measurable. If $u$ is obtained by application of the von Neumann's selection theorem ([3], III.22) it is $\hat{\mathcal{A}}-\mathfrak{B}\left(E_{s}^{\prime}\right)$ measurable. But there exists a Polish topology $\mathcal{C}$ on $E^{\prime}$ finer than $\sigma\left(E^{\prime}, E\right)$ whose Borel tribe is still $\mathcal{B}\left(E_{s}^{\prime}\right)$. Hence there exists $u^{\prime}: \Omega \rightarrow E_{s}^{\prime}$ which is $\mathcal{A}-\mathscr{B}\left(E_{s}^{\prime}\right)$ measurable and equal a.e. to $u$.

2) Let $\Gamma \in \operatorname{CRS}\left(\hat{\mathfrak{A}} ; V_{n}^{0}\right)$, then for every open set $U\{\omega \mid \Gamma(\omega) \cap U \neq \emptyset\}$ belongs to $\hat{\mathfrak{A}}$. It follows from [3], III.2 that $\Gamma$ is measurable with respect to $\hat{\mathcal{A}}$ and the 
Borel tribe of the Hausdorff metric on the set of closed subsets of $V_{n}^{0}$, Hence there exists $\Gamma^{\prime}$ which is $\mathcal{A}$-measurable and equal a.e. to $\Gamma$.

3) Let $\Gamma \in \operatorname{CCRS}\left(\hat{\mathfrak{A}} ; E^{\prime}\right)$. Then $\left(\omega, x^{\prime}\right) \mapsto \delta\left(x^{\prime} \mid \Gamma(\omega)\right)$ is a convex normal integrand on $(\Omega, \hat{\mathfrak{A}}) \times E_{s}^{\prime}$. So there exists a sequence $\left(v_{n}, r_{n}\right)$ of $\hat{\mathfrak{A}}$-measurable selection of $\omega \mapsto$ epi $\delta^{*}(\cdot \mid \Gamma(\omega))$ such that

$$
\text { epi } \delta^{*}(\cdot \mid \Gamma(\omega))=\operatorname{cl}\left\{\left(v_{n}(\omega), r_{n}(\omega)\right) \mid n \in N\right\} .
$$

As $\left(v_{n}, r_{n}\right)$ takes its values in $E \times R$ there exists $\mathcal{A}$-measurable functions $\left(\bar{v}_{n}, \bar{r}_{n}\right)$ such that $\left(\bar{v}_{n}, \bar{r}_{n}\right)=\left(v_{n}, r_{n}\right)$ a.e.

Then $\Gamma^{\prime}(\omega)=\cap\left\{x^{\prime} \mid\left\langle x^{\prime}, \bar{v}_{n}(\omega)\right\rangle \leqslant \bar{r}_{n}(\omega)\right\}$ has its graph in $\mathcal{A} \otimes \mathscr{B}_{(}\left(E_{s}^{\prime}\right)$ and is equal to $\Gamma$ a.e.

\section{REFERENCES}

[1] J. M. Brsmut, Intégrales convexes et probabilités, J. of Math. An. and Appl., 42, no. 3 (1973), pp. 639-673.

[2] C. Castaing, Oompacité et inf-équicontinuité dans certains espaces de Köthe-Orlicz, Séminaire d'Analyse Convexe, Montpellier, 1979, Exposé no. 6.

[3] C. Castaing - M. ValadieR, Convex analysis and measurable multifunctions, Lecture Notes in Math. no. 580 (1977), Springer-Verlag.

[4] J. P. Daures, Version multivoque du théorème de Doob, Ann. Inst. H. Poincaré, 9, no. 2 (1973), pp. 167-176.

[5] E. B. DYNKIN - I. V. EVSTIGNEEv, Regular conditional expectations of correspondences, Theory of proba. and its. appl., 21 (1976), pp. 325-338.

[6] F. HIAI - H. UMEGAKI, Integrals, conditional expectations and martingales of multivalued functions, J. of Multivariate an., 7 (1977), pp. 149-182.

[7] J. Neveu, Convergence presque-süre de martingales multivoques, Ann. Inst. H. Poincaré, 8, no. 1 (1972), pp. 1.7.

[8] C. OLECH, Existence theory in optimal problems. The underlying ideas, in International Conferences on Differential Equations, Academic Press (1975), pp. 612-635.

[9] M. F. SAINTE-BEUve, Some topological properties of vector measures with bounded varia. tions and its applications, Annali di Mat. Pura ed Appl., 116 (1978), pp. 317-379.

[10] M. VALADIER, Contribution à l'analyse convexe, Thèse, Paris (1970).

[11] M. VALADTER, Multiapplications mesurables à valeurs convexes compactes, J. Math. pures et appl., 50 (1971), pp. 265-297.

[12] M. VALADIER, Espérance conditionnelle d'un convexe fermé aléatoire, C. R. Acad. Sc., 273 (1971), pp. 1265-1267; et Séminaire d'Analyse Convexe, Montpellier, 1972, Exposé no. 1.

[13] M. VALAdrer, Fermeture, régularisée sci et bipolaire bagues, C. R. Acad. Sc., 284 (1977), pp. 1025-1027; ot Séminaire d'Analyse Convexe, Montpellier, 1977, Exposé no. 6.

[14] M. VALADIER, Sur l'espóranoe eonditionnelle multivoque, Séminaire d'Analyse Convexe, Montpellier, 1978, Exposé no. 9.

[15] B. VAN Cutswa, Eléments aléatoires d̀ valeurs convexes compacts, Thèse, Grenoble (1971).

[16] J. L. Doob, Stochastic process, John Wiley \& Sons (1953).

[17] M. Loeve, Probability theory, Van Nostrand (1963). 